\title{
Use of HOMA-IR to diagnose non-alcoholic fatty liver disease: a population-based and inter-laboratory study
}

\author{
Elina Isokuortti ${ }^{1,2}$ (D) You Zhou ${ }^{3,4} \cdot$ Markku Peltonen $^{5} \cdot$ Elisabetta Bugianesi $^{6}$ • \\ Karine Clement $^{7,8}$ • Dominique Bonnefont-Rousselot ${ }^{\text {9,10,11 }}$ - Jean-Marc Lacorte ${ }^{\text {7,8,12 }}$ • \\ Amalia Gastaldelli ${ }^{13}$ • Detlef Schuppan ${ }^{14}$ • Jörn M. Schattenberg ${ }^{15}$. \\ Antti Hakkarainen $^{16}$ • Nina Lundbom ${ }^{16}$ - Pekka Jousilahti ${ }^{5}$ Satu Männistö ${ }^{5}$. \\ Sirkka Keinänen-Kiukaanniemi ${ }^{17}$ - Juha Saltevo ${ }^{18}$ • Quentin M. Anstee ${ }^{19,20}$. \\ Hannele Yki-Järvinen ${ }^{1,2}$
}

Received: 6 March 2017 / Accepted: 15 May 2017 / Published online: 29 June 2017

(C) Springer-Verlag GmbH Germany 2017

\begin{abstract}
Aims/hypothesis Recent European guidelines for nonalcoholic fatty liver disease (NAFLD) call for reference values for HOMA-IR. In this study, we aimed to determine: (1) the upper limit of normal HOMA-IR in two population-based cohorts; (2) the HOMA-IR corresponding to NAFLD; (3)
\end{abstract}

Electronic supplementary material The online version of this article (doi:10.1007/s00125-017-4340-1) contains peer-reviewed but unedited supplementary material, which is available to authorised users.

Elina Isokuortti

elina.isokuortti@helsinki.fi

1 Minerva Foundation Institute for Medical Research, Biomedicum Helsinki 2 U, Tukholmankatu 8, FIN - 00290 Helsinki, Finland

2 Department of Medicine, University of Helsinki and Helsinki University Hospital, Helsinki, Finland

3 Systems Immunity University Research Institute, Cardiff University, Cardiff, UK

4 Division of Infection and Immunity, School of Medicine, Cardiff University, Cardiff, UK

5 National Institute for Health and Welfare, Helsinki, Finland

6 Division of Gastroenterology, Department of Medical Sciences, University of Torino, Torino, Italy

7 Institute of Cardiometabolism and Nutrition (ICAN), Pitié-Salpêtrière Hospital, Paris, France

8 Sorbonne Université, UPMC University Paris 06, UMR_S 1166, Inserm, Paris, France

9 Department of Metabolic Biochemistry, La Pitié Salpêtrière-Charles Foix University Hospital (AP-HP), Paris, France

10 Department of Biochemistry, Faculty of Pharmacy, Paris Descartes University, Paris, France the effect of sex and PNPLA3 genotype at rs738409 on HOMA-IR; and (4) inter-laboratory variations in HOMA-IR. Methods We identified healthy individuals in two population-based cohorts (FINRISK 2007 [ $n=5024]$ and the Programme for Prevention of Type 2 Diabetes in Finland [FIN-D2D; $n=2849]$ ) to define the upper 95th

11 CNRS UMR8258 - Inserm U1022, Faculty of Pharmacy, Paris Descartes University, Paris, France

12 Department of Endocrine and Oncological Biochemistry, La Pitié Salpêtrière-Charles Foix University Hospital (AP-HP), Paris, France

13 Cardiometabolic Risk Laboratory, Institute of Clinical Physiology, CNR, Pisa, Italy

14 Institute of Translational Immunology, Research Center of Immune Therapy, University Medical Centre, Johannes Gutenberg University Mainz, Mainz, Germany

15 Department of Medicine I, University Medical Centre, Johannes Gutenberg University Mainz, Mainz, Germany

16 Helsinki Medical Imaging Center, University of Helsinki and Helsinki University Hospital, Helsinki, Finland

17 Institute of Health Sciences, University of Oulu, Oulu, Finland

18 Department of Medicine, Central Finland Central Hospital, Jyväskylä, Finland

19 Institute of Cellular Medicine, The Medical School, Newcastle University, Newcastle upon Tyne, UK

20 Liver Unit, Newcastle Upon Tyne Hospitals NHS Trust, Freeman Hospital, Newcastle upon Tyne, UK 
percentile of HOMA-IR. Non-obese individuals with normal fasting glucose levels, no excessive alcohol use, no known diseases and no use of any drugs were considered healthy. The optimal HOMA-IR cut-off for NAFLD (liver fat $\geq 5.56 \%$, based on the Dallas Heart Study) was determined in 368 non-diabetic individuals $(35 \%$ with NAFLD), whose liver fat was measured using proton magnetic resonance spectroscopy $\left({ }^{1} \mathrm{H}-\mathrm{MRS}\right)$. Samples from ten individuals were simultaneously analysed for HOMA-IR in seven European laboratories.

Results The upper 95th percentiles of HOMA-IR were 1.9 and 2.0 in healthy individuals in the FINRISK $(n=1167)$ and FIN-D2D $(n=459)$ cohorts. Sex or PNPLA3 genotype did not influence these values. The optimal HOMA-IR cutoff for NAFLD was 1.9 (sensitivity $87 \%$, specificity $79 \%$ ). A HOMA-IR of 2.0 corresponded to normal liver fat $\left(<5.56 \%\right.$ on $\left.{ }^{1} \mathrm{H}-\mathrm{MRS}\right)$ in linear regression analysis. The 2.0 HOMA-IR measured in Helsinki corresponded to 1.3, $1.6,1.8,1.8,2.0$ and 2.1 in six other laboratories. The inter-laboratory CV\% of HOMA-IR was $25 \%$ due to inter-assay variation in insulin $(25 \%)$ rather than glucose (5\%) measurements.

Conclusions/interpretation The upper limit of HOMA-IR in population-based cohorts closely corresponds to that of normal liver fat. Standardisation of insulin assays would be the first step towards definition of normal values for HOMA-IR.

Keywords Insulin $\cdot$ Liver fat $\cdot$ NAFLD $\cdot$ PNPLA3 · Reference values

$\begin{array}{ll}\text { Abbreviations } \\ \text { ALT } & \begin{array}{l}\text { Alanine aminotransferase } \\ \text { Aspartate aminotransferase }\end{array} \\ \text { AUROC } & \begin{array}{l}\text { Area under the receiver operating } \\ \text { characteristic (curve) }\end{array} \\ \text { DHS } & \begin{array}{l}\text { Dallas Heart Study } \\ \text { Dietary Lifestyle and Genetic Determinants } \\ \text { of the Development of Obesity and Metabolic }\end{array} \\ & \begin{array}{l}\text { Syndrome study } \\ \text { FIN-D2D }\end{array} \\ \begin{array}{l}\text { Programme for Prevention of Type 2 } \\ \text { Diabetes in Finland } \\ \text { GGT }\end{array} & \begin{array}{l}\gamma \text {-Glutamyltransferase } \\ \text { Proton magnetic resonance spectroscopy }\end{array} \\ \text { NAFLD } & \begin{array}{l}\text { Non-alcoholic fatty liver disease } \\ \text { Negative predictive value }\end{array} \\ \text { NPV } & \begin{array}{l}\text { Patatin-like phospholipase } \\ \text { PNPLA3ain-containing protein } 3\end{array} \\ \text { PPV } & \begin{array}{l}\text { Positive predictive value } \\ \text { Receiver operating characteristic }\end{array} \\ \text { ROC } & \end{array}$

\section{Introduction}

Insulin resistance in individuals with non-alcoholic fatty liver disease (NAFLD) is characterised by reduced whole body, hepatic and adipose tissue insulin sensitivity [1,2]. The liver, once insulin resistant, overproduces glucose that stimulates insulin secretion, resulting in mild hyperglycaemia and hyperinsulinaemia. Therefore, the product of fasting glucose and fasting insulin divided by a constant (i.e. HOMA-IR) [3] is a good surrogate for insulin sensitivity in non-diabetic individuals [3-5]. Once the glucose concentration reaches the diagnostic threshold for type 2 diabetes, the insulin concentration starts to decline relative to glucose [6-9] and HOMA-IR no longer exclusively reflects insulin sensitivity.

A recent joint European practice guideline for NAFLD [10] concluded: 'HOMA-IR provides a surrogate estimate of insulin resistance in persons without diabetes and can therefore be recommended, provided proper reference values have been established.' A reference value can be defined as the mean + 2 SDs for normally distributed variables or the 95 th percentile for non-normally distributed variables of a population-based sample [11, 12]. Definition of health then becomes dependent on the underlying population. This is particularly relevant for HOMA-IR, as obesity is highly prevalent and perhaps the single most important cause of variation in insulin [13]. Thus, for HOMA-IR, it would seem wiser to use reference values derived from healthy individuals, although definitions of health can also vary [14-16]. Pre-analytical causes of variation should also be considered [3, 4], and the inter-assay variation of insulin [17] and glucose should be known.

Normal liver fat content, measured using proton magnetic resonance spectroscopy $\left({ }^{1} \mathrm{H}-\mathrm{MRS}\right)$, was determined in the population-based Dallas Heart Study (DHS) [18]. In healthy individuals (BMI $<25 \mathrm{~kg} / \mathrm{m}^{2}$, no diabetes, normal fasting glucose levels, low alcohol consumption, no known liver disease or risk factors for liver disease and normal alanine aminotransferase [ALT] levels; $n=345$ ), the 95th percentile for liver fat content was $5.56 \%$. It is unknown how HOMA-IR relates to this amount of liver fat and whether this definition of normal liver fat reflects what normal liver fat is elsewhere. The I148M variant of the gene encoding patatin-like phospholipase domain-containing protein 3 (PNPLA3) (rs738409 c.444 C $>$ G, p.I148M) has a prevalence of 30-50\% [19]. It increases the risk of NAFLD, but not of insulin resistance [20]. The impact of this gene variant on reference values for HOMAIR has not been studied.

In the present study, we aimed to determine: (1) the upper 95th percentile of HOMA-IR in two population-based cohorts; (2) the HOMA-IR that best distinguishes between NAFLD and normal liver fat content, as quantified by ${ }^{1} \mathrm{H}-$ MRS in a cohort of non-diabetic individuals; (3) whether sex or the PNPLA3 genotype at rs 738409 influences reference values for HOMA-IR; and (4) the inter-laboratory variation in 
HOMA-IR among European centres participating in the Elucidating Pathways of Steatohepatitis (EPoS) consortium (www.epos-nafld.eu).

\section{Methods}

\section{Study designs}

Population-based cohorts for the determination of normal HOMA-IR To determine normal HOMA-IR, we studied nonpregnant adults in two population-based cohorts: the National FINRISK 2007/ Dietary Lifestyle and Genetic Determinants of the Development of Obesity and Metabolic Syndrome study (DILGOM) study ( $n=5024)$, conducted by the National Institute for Health and Welfare in Finland between January and July 2007 [21]; and the Programme for Prevention of Type 2 Diabetes in Finland (FIN-D2D) ( $n=2849$ ), conducted between October and December 2007 [22] (see electronic supplementary material [ESM] Methods and ESM Fig. 1). The definition of healthy was as in the population-based DHS [18]: (1) alcohol use <30 g/day in men and $<20 \mathrm{~g} /$ day in women; (2) no diabetes, based on history and normal fasting plasma glucose levels $(<6.1 \mathrm{mmol} / \mathrm{l})$; (3) $\mathrm{BMI}<25 \mathrm{~kg} / \mathrm{m}^{2}$; (4) no regular use of drugs; and (5) no clinical or biochemical evidence of liver or other disease, as defined by history and biochemical examinations.

Liver fat cohort Participants for the liver fat cohort were recruited using newspaper advertisements, by contacting occupational health services and from individuals referred to the Department of Gastroenterology, Helsinki University Hospital (Helsinki, Finland), because of chronically elevated serum transaminase concentrations using the following inclusion criteria: (1) age 18-75 years; (2) no known acute or chronic disease except obesity, hypertension or NAFLD based on medical history, physical examination, standard laboratory tests and ECG; (3) non-diabetic based on a fasting plasma glucose level of $\leq 6.9 \mathrm{mmol} / \mathrm{l}$; and (4) alcohol consumption of $\leq 20 \mathrm{~g}$ per day in women and $\leq 30 \mathrm{~g}$ in men [23]. Study physicians assessed alcohol intake using the same questionnaire as in the population-based studies. Exclusion criteria included: (1) pregnancy; (2) serologic evidence of hepatitis $\mathrm{B} / \mathrm{C}$ or autoimmune hepatitis; (3) clinical signs or symptoms of inborn errors of metabolism; (4) a history of predisposition to toxins; (5) use of drugs associated with liver steatosis; and (6) use of antihypertensive drugs or other drugs possibly influencing glucose metabolism. The study protocol was approved by the ethics committee of the Helsinki University Central Hospital and was carried out in accordance with the Declaration of Helsinki. Each participant provided written informed consent.
Inter-laboratory variation in insulin assays Ten nondiabetic individuals covering a wide range of insulin sensitivities were recruited. The participants were healthy based on medical history, physical examination and standard laboratory tests, but eight of them were overweight or obese (BMI $\geq 25 \mathrm{~kg} / \mathrm{m}^{2}$ ). Blood was drawn in Helsinki after a $12 \mathrm{~h}$ fast for measurement of fasting insulin, glucose, HDL-cholesterol, LDL-cholesterol, total cholesterol, triacylglycerol, aspartate aminotransferase (AST), ALT, $\gamma$-glutamyltransferase (GGT), ferritin and albumin. Measurements of laboratory variables other than insulin or glucose were performed for comparison to estimate their inter-assay CVs. The fresh samples were analysed immediately in Helsinki. To study the effect of freezing, another set of samples were immediately frozen to $-80^{\circ} \mathrm{C}$ and then melted and assayed on the same day in Helsinki. To study the effect of time, a third set of samples were frozen to $-80^{\circ} \mathrm{C}$ and assayed after 2 weeks in Helsinki. At this same time point, six additional sets of samples, which had been shipped in dry ice, were assayed in Newcastle (UK), Paris (France), Pisa (clinical and research laboratories; Italy), Torino (Italy) and Mainz (Germany). The study protocol was approved by the ethics committee of the Helsinki University Central Hospital and was carried out in accordance with the Declaration of Helsinki. Each participant provided written informed consent.

\section{Biochemical measurements}

FINRISK/DILGOM and FIN-D2D Biochemical assays were performed in the Laboratory of Analytical Biochemistry of the Institute of Health and Welfare (Helsinki, Finland) using an Architect ci8200 analyser (Abbott Laboratories, Abbott Park, IL, USA). Plasma glucose was determined using the hexokinase method (Abbott Laboratories) and serum insulin using a chemiluminescent microparticle immunoassay (Abbott Laboratories). Serum total cholesterol, HDL-cholesterol and triacylglycerol concentrations were measured using enzymatic kits (Abbott Laboratories), and the LDL-cholesterol concentration was calculated using the Friedewald formula [24]. Total cholesterol was measured using the CHOD-PAP assay (Abbott Laboratories). Samples were stored at $-80^{\circ} \mathrm{C}$ before analysis. In the FIN-D2D study, $\mathrm{HbA}_{1 \mathrm{c}}$ was measured using an immunoturbidimetric method (Abbott Laboratories), and serum ALT, AST and GGT concentrations were measured using International Federation of Clinical Chemistry photometric methods (Abbott Laboratories). In the liver fat cohort, plasma glucose was measured using the hexokinase method in an autoanalyser (Roche Diagnostics Hitachi 917; Hitachi, Tokyo, Japan) and serum insulin was measured in fresh serum samples using a time-resolved fluoroimmunoassay with AutoDELFIA kits (Wallac, Turku, Finland). $\mathrm{HbA}_{1 \mathrm{c}}$ was measured using HPLC using the fully automated analyser system 
(Bio-Rad, Richmond, CA, USA). Serum triacylglycerol, total cholesterol, LDL-cholesterol and HDL-cholesterol concentrations were measured with enzymatic kits from Roche Diagnostics using an autoanalyser (Roche Diagnostics Hitachi 917; Hitachi). Serum ALT, AST and GGT activities were determined as recommended by the European Committee for Clinical Laboratory Standards using the Roche Diagnostics Hitachi 917 (Hitachi). HOMA-IR was calculated as described by Matthews et al [3]. The methods used by the seven participating centres for HOMA-IR and the other laboratory variables are shown in ESM Methods.

\section{Genotyping of PNPLA3 at rs738409}

FINRISK/DILGOM The PNPLA3 genotype was determined from 1000G imputed genome-wide association study data consisting of three subsets genotyped using the Illumina HumanCoreExome, Illumina OmniExpress and Illumina 610K (Illumina, San Diego, CA, USA).

FIN-D2D Genomic DNA was extracted from whole blood using automated Chemagen DNA extraction equipment (PerkinElmer, Waltham, MA, USA) or a QIAamp DNA Blood Maxi Kit (Qiagen, Hilden, Germany) following the protocol of the kit with slight modifications. Genotyping was performed using a TaqMan assay (Applied Biosystems, Paisley, UK).

Liver fat cohort DNA was isolated from whole blood and the PNPLA3 genotype at rs738409 was determined as previously described using a TaqMan assay (Applied Biosystems) [25].

\section{Measurement of liver fat content by ${ }^{1}$ H-MRS}

Liver fat was measured by using ${ }^{1} \mathrm{H}-\mathrm{MRS}$ as previously described [23]. Liver fat content was expressed as a mass fraction in percentage units [23]. NAFLD was defined as in the DHS (liver fat $\geq 5.56 \%$ by ${ }^{1} \mathrm{H}-\mathrm{MRS}$ ) [18].

\section{Other measurements}

In all cohorts, body weight, height, BMI and waist and hip circumferences were measured as previously described [23, 26, 27].

\section{Statistical analysis}

Distribution of continuous variables was analysed for normality using the Kolmogorov-Smirnov test. Data are shown as means \pm SD for normally distributed data and as medians (25$75 \%$ ) for non-normally distributed data. To compare characteristics among groups, the unpaired $t$ test and the MannWhitney $U$ test were used for continuous variables, and
Fisher's exact test and the $\chi^{2}$ test were used for categorical variables, where appropriate. Logarithmic transformation was performed for non-normally distributed data if needed. Correlation analyses were performed using Pearson's correlation coefficient.

Healthy individuals in the FINRISK/DILGOM $(n=1167)$ and FIN-D2D $(n=459)$ cohorts were identified. HOMA-IR was not normally distributed, and therefore the 95 th percentile $(90 \% \mathrm{CI})$ rather than the mean $+2 \mathrm{SD}$ was used to determine the upper reference value for HOMA-IR [12]. After $\log _{2}$ transformation, HOMA-IR values were adjusted in a generalised linear model, using age and BMI as covariates.

We used two methods to identify a cut-off value of HOMAIR for NAFLD. First, we calculated the HOMA-IR value that corresponded to the normal liver fat content based on the DHS (liver fat $<5.56 \%$ [18]) using linear regression analysis. We tested whether the slopes and intercepts in linear regression analysis differed between men and women, and carriers and non-carriers of the PNPLA3 I148M variant. The 95th percentile was used to define normal liver fat content in healthy individuals in the liver fat cohort, as in the DHS [18]. Second, we determined the receiver operating characteristic (ROC) curve to calculate the area under the ROC curve (AUROC [95\% CI]). The Youden index [28] was used to identify the optimal cut-off of HOMA-IR. For this, individuals were randomly divided into discovery (two-thirds of the individuals) and validation (one-third) groups. The discovery group was used to determine the ROC curve for HOMA-IR. The validation group and all individuals were used for validation. For additional validation, we generated 1000 random sets of samples and used the bootstrap method to validate the model in the sample sets. The AUROC of each set was estimated, and the average of these estimates provided the overall prediction accuracy of the model. Power analysis was conducted to estimate the appropriate sample size for correlation analysis and ROC analysis. To detect a correlation coefficient of 0.2 between HOMA-IR and liver fat content with a power of 0.8 , a sample size of at least 193 was required. By setting the ratio of sample sizes between negative and positive groups at 2 , at least 23 cases and 46 control participants were needed to reach a statistical power of 0.8 to detect the minimum AUROC of 0.7 .

The inter-laboratory CVs of fasting insulin, glucose, HOMA-IR, lipids, liver enzymes, ferritin and albumin among laboratories were calculated. Linear regression analyses were performed to compare insulin, glucose and HOMA-IR measurements in Helsinki to those in other centres. The HOMAIR in each centre corresponding to the upper limit of normal HOMA-IR in Helsinki was defined from linear regression equations.

We considered a $p$ value of $<0.05$ to be statistically significant. Calculations were made using $\mathrm{R}$ Project version 3.1.1 (www.r-project.org, Vienna, Austria) and GraphPad Prism 
version 6.00 for Mac OS X (GraphPad Software, San Diego, CA, USA).

\section{Results}

\section{Reference values for HOMA-IR in two population-based cohorts}

Characteristics of the healthy individuals in the two population-based cohorts ( $n=1167$ in FINRISK/DILGOM, $n=459$ in FIN-D2D) are shown in Table 1. Characteristics of these individuals subgrouped based on their PNPLA3 genotype at rs738409 are shown in ESM Table 1. The upper limit of normal (95th percentile [90\% CI]) HOMA-IR was 1.9 (1.8, $2.0)$ in the FINRISK/DILGOM cohort and $2.0(1.9,2.2)$ in the FIN-D2D cohort (Fig. 1).

There was no sex difference among HOMA-IRs in either cohort (Fig. 1). Since there were slight differences in age and BMI between men and women in the two studies (Table 1) we also calculated age- and BMI-adjusted HOMA-IRs, which were very similar to the unadjusted values (Table 1). The PNPLA3 genotype did not influence HOMA-IR in either cohort (Fig. 1).

The 95th percentile of serum ALT in the FIN-D2D cohort was $31 \mathrm{U} / 1$ in women and $43 \mathrm{U} / 1$ in men. In the FINRISK/ DILGOM cohort aged 25-74 years, age weakly inversely correlated with HOMA-IR $(\rho=-0.16, p<0.001)$. No significant relationship was observed between age and HOMA-IR in the FIN-D2D cohort aged 45-74 years $(\rho=0.06, p=0.21)$.

\section{Relationship between HOMA-IR and liver fat content}

Characteristics of the non-diabetic individuals in the liver fat cohort $(n=368)$ are shown in Table 1 . Of them, $35 \%$ had NAFLD as evaluated by ${ }^{1} \mathrm{H}-\mathrm{MRS}$. Liver fat percentage positively correlated with HOMA-IR $(r=0.67, p<0.001)$ (Fig. 2a). Normal liver fat, defined as in the DHS $(<5.56 \%)$, corresponded to a HOMA-IR of 2.0 (95\% CI 1.9, 2.1) (Fig. 2a) in non-diabetic individuals, with a HOMA-IR of $1.9(1.8,2.1)$ in women and $2.1(1.9,2.2)$ in men $(p=0.29)$. The HOMA-IR corresponding to the normal liver fat content $(<5.56 \%)$ was significantly higher in non-carriers $(2.1$ [2.0, 2.2]) than carriers $(1.8[1.6,1.9], p=0.007)$ of the PNPLA3 I148M variant (Fig. 2b) (i.e. the variant allele carriers had a higher liver fat content for any given HOMA-IR than noncarriers). The upper 95th percentile for liver fat in the 96 healthy individuals was $5.9 \%$.

The discovery and validation groups for defining the HOMA-IR cut-off for NAFLD were similar with respect to clinical and biochemical characteristics (ESM Table 2). The AUROC for HOMA-IR was 0.88 (95\% CI 0.84, 0.92) in the discovery group (Fig. 3). The optimal HOMA-IR cut-off for
NAFLD, based on the Youden index, the point of optimal sensitivity and specificity, was 1.9. This cut-off had a sensitivity of $87 \%$, specificity of $79 \%$, negative predictive value (NPV) of $92 \%$ and positive predictive value (PPV) of $67 \%$. The results were similar for the validation group (AUROC $0.80[0.70,0.88]$, sensitivity $68 \%$, specificity $82 \%$, NPV $81 \%$ and PPV 70\%) and for all individuals (AUROC 0.85 [0.80, 0.89] sensitivity $80 \%$, specificity $80 \%$, NPV $88 \%$ and PPV 68\%) (Fig. 3). The AUROC for bootstrap samples was $0.88(0.82,0.92)$ and the overall estimate of optimism was 0.00079 . Neither sex $(p=0.22)$ nor PNPLA3 genotype $(p=0.18)$ significantly influenced the AUROC.

\section{Inter-laboratory variation in insulin assays and HOMA-IR}

The ten individuals (three men, seven women) recruited to investigate inter-laboratory variations ranged in age from 22 to 62 years and in BMI from 21.3 to $42.4 \mathrm{~kg} / \mathrm{m}^{2}$. Among the seven laboratories, the mean values ranged from 18.0 to $91.2 \mathrm{pmol} / 1$ for insulin, from 4.7 to $6.1 \mathrm{mmol} / 1$ for glucose and from 0.69 to 4.0 for HOMA-IR. Freezing and thawing the serum on the same day had no impact on fasting insulin $(52.8 \pm 28.8$ vs $54.0 \pm 29.4 \mathrm{pmol} / 1, p=0.077)$. Serum insulin concentrations decreased over time when stored at $-80^{\circ} \mathrm{C}$ degrees for 2 weeks $(54.0 \pm 29.4$ vs $45.6 \pm 25.8 \mathrm{pmol} / 1$, $p=0.005)$.

The CV of fasting insulin measured in the seven participating laboratories after 2 weeks of storage at $-80^{\circ} \mathrm{C}$ averaged $25.4 \%$. The CV of fasting glucose was significantly lower and averaged $4.6 \%$. The CV of HOMA-IR was $25.0 \%$. The HOMA-IR value of 2.0, as measured in Helsinki, corresponded to HOMA-IRs of 1.3, 1.6, 1.8, 1.8, 2.0 and 2.1 in the six other centres (Fig. 4). The relationships between insulin and glucose measurements in Helsinki vs the other centres are shown in ESM Fig. 2, 3.

The inter-laboratory CVs for the other analytes were as follows: total cholesterol 7.4\%, LDL-cholesterol $12.8 \%$, HDL-cholesterol 7.0\%, triacylglycerol $8.3 \%$, AST $11.7 \%$, ALT $11.6 \%$, GGT $11.3 \%$, ferritin $19.1 \%$ and albumin $7.7 \%$. All of these CVs, with the exception of ferritin, were significantly lower than the $\mathrm{CV}$ for fasting insulin $(p<0.01)$.

\section{Discussion}

The present studies were undertaken to determine whether a single value of HOMA-IR could be used to clearly identify individuals with NAFLD, and how HOMA-IRs determined by different laboratories in European countries compare with each other. In two population-based cohorts, the upper limits of normal HOMA-IR were 1.9 and 2.0. In individuals whose liver fat content was determined using ${ }^{1} \mathrm{H}-\mathrm{MRS}$, a HOMA-IR 


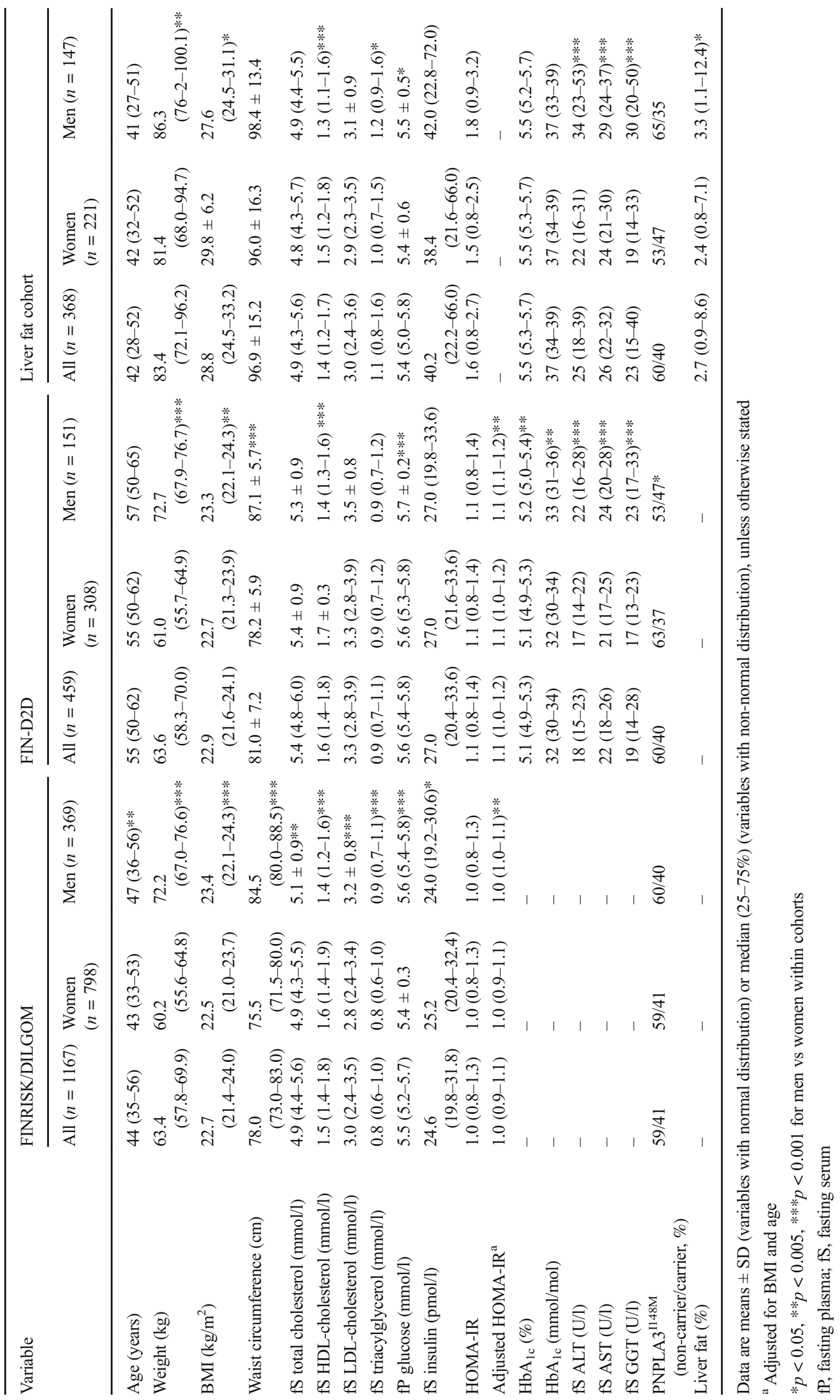


Fig. 1 Percentiles (90\% CI) of HOMA-IR in healthy men (triangles and dotted/dashed lines), women (squares and dashed lines) and all individuals (circles and solid lines) in the population-based FINRISK/ DILGOM (a) and FIN-D2D cohorts (b), and in I148M variant non-carriers (PNPLA3 ${ }^{\mathrm{I} 148 \mathrm{II}}$; squares and dashed lines) and carriers (PNPLA3 ${ }^{\mathrm{I1} 48 \mathrm{IM} / \mathrm{MM} \text {; }}$ triangles and dotted/dashed lines) and all individuals (circles and solid lines) in the populationbased FINRISK/DILGOM (c) and FIN-D2D cohorts (d)
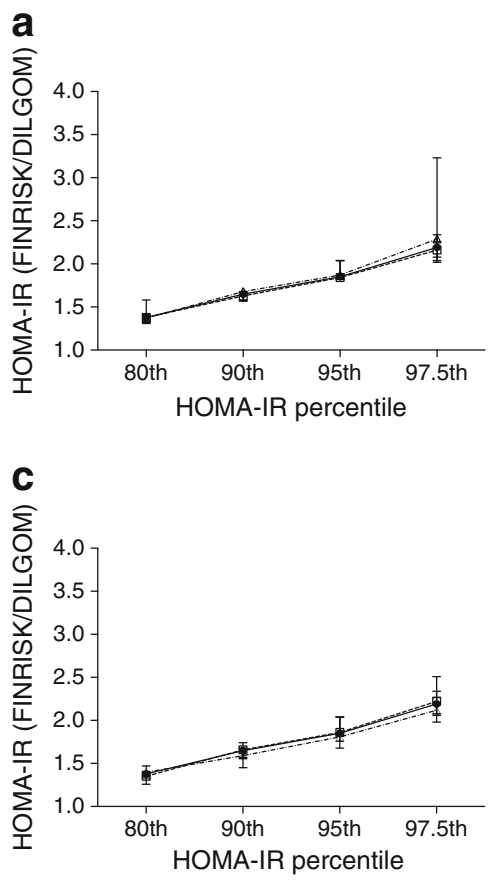

b

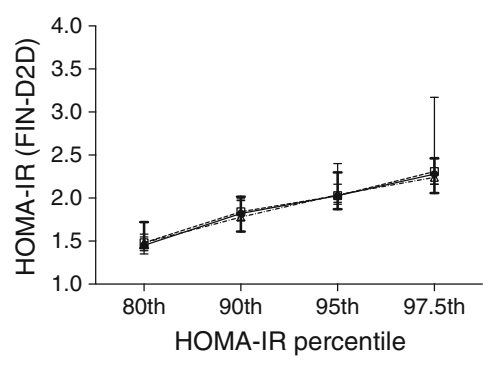

d

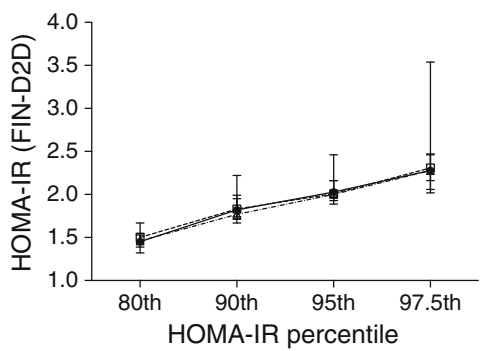

cut-off of 1.9 was optimal for diagnosing NAFLD based on the Youden index. A HOMA-IR of 2.0 corresponded to the upper limit of normal liver fat content of $5.56 \%$, as defined in the DHS. A HOMA-IR value of 2.0 corresponded to HOMAIRs between 1.3 and 2.1 in six other laboratories, with an interlaboratory CV of $25 \%$. These data show that the upper limit of normal HOMA-IR closely corresponds to the upper limit of liver fat defined as in the DHS, and that there is large interlaboratory variation in insulin measurements.

The upper limit of a reference value is usually defined in population-based samples of healthy individuals as the mean + 2 SD in normally distributed samples and as the 95th percentile in non-normally distributed samples [12]. In this study, the 95th percentiles were 1.9 and 2.0 in the healthy individuals of two population-based cohorts. Three previous studies have been performed in healthy individuals. These studies were

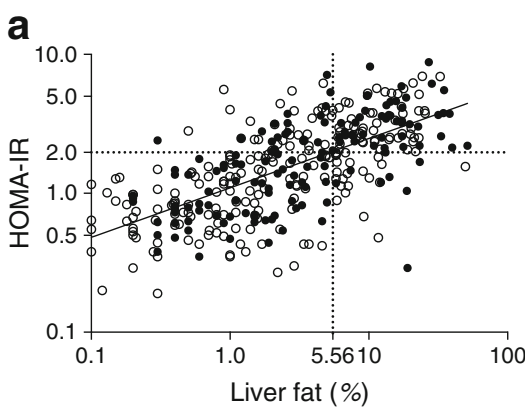

Fig. 2 Relationship between liver fat measured by ${ }^{1} \mathrm{H}-\mathrm{MRS}\left(\log _{10}\right)$ and HOMA-IR $\left(\log _{10}\right)$. (a) The relationship was similar (slopes $p=0.79$, elevations $p=0.75)$ in men (black circles) $(r=0.67, p<0.001)$ and in women (white circles) $(r=0.66, p<0.001)$. The HOMA-IR corresponding to normal liver fat $(<5.56 \%)$, as defined in the DHS [18], was 2.0. (b) There was a significant difference in the intercepts of the regression lines smaller (161 Japanese, 161 Italian and 312 Brazilian individuals) than the present study (459-1167 Finnish individuals) [14-16]. In the Japanese study [14], the 90th percentile of HOMA-IR was 1.7, which is comparable with that found in the present study. In the Italian study, however, the participants were not healthy as they included diabetic and hypertensive individuals. The 80th percentile of HOMA-IR was 2.77 [16]. This study used a non-specific RIA from Linco Research (St. Charles, MO, USA), which has produced the highest insulin concentrations of several insulin assays tested $[17,29]$. Similarly, the Brazilian study also used this RIA, and the 90th percentile of HOMA-IR was equally high (2.71) [15]. Thus, the higher HOMA-IR in these studies compared with the present study could be due to the inclusion of diabetic and hypertensive individuals in the Italian study, and to the use of an RIA that is no longer used in most laboratories [17].

b

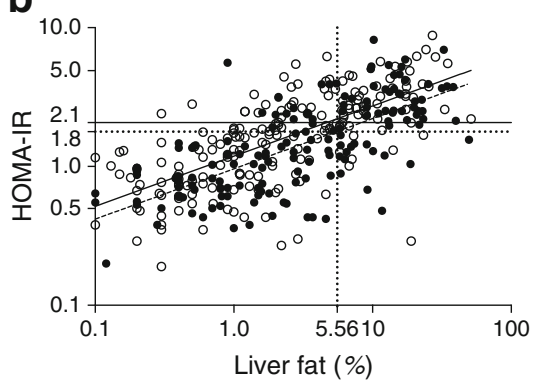

$(p=0.007)$ between carriers (PNPLA $\left.3^{\mathrm{I148 \textrm {IM }} / \mathrm{MM}}\right)(r=0.69, p<0.001)$ (black circles) and non-carriers (PNPLA3 $\left.{ }^{\mathrm{I1} 48 \mathrm{II}}\right)(r=0.68, p<0.001)$ (white circles) of the I148M variant, showing that HOMA-IR was lower for any given liver fat content in carriers than non-carriers. No significant difference between the slopes was observed $(p=0.99)$ 


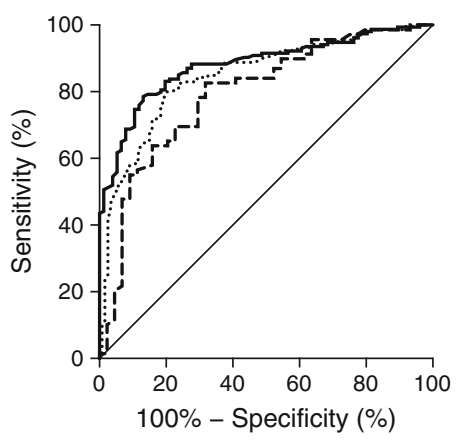

Fig. 3 AUROC for HOMA-IR and NAFLD. The AUROC for HOMAIR was 0.88 (95\% CI $0.84,0.92)$ in the discovery group (solid line), 0.80 $(0.70,0.88)$ in the validation group (dashed line) and $0.85(0.80,0.89)$ in all individuals (dotted line)

We found no significant differences in HOMA-IR percentiles between men and women among the healthy individuals in either population-based cohort (Fig. 1). The men were, however, slightly more obese and older than the women, and therefore we also calculated age- and BMI-adjusted HOMAIRs. After adjustment, men had slightly higher HOMA-IRs than women in both studies, but the differences in absolute units were trivial (0.02 in FINRISK/DILGOM and 0.05 in the FIN-D2D study; Table 1). Previous population-based studies including healthy individuals have not reported HOMA-IRs separately for men and women [14-16].

In keeping with the 95th percentile in healthy individuals in the population-based cohorts, we found a HOMA-IR of 1.9 to best distinguish non-diabetic individuals with and without NAFLD. This value is similar to that found in 204 Brazilian individuals [30]. As in the present study, in ROC analysis, a HOMA-IR of 2.0 (AUROC 0.84) best distinguished between NAFLD and non-NAFLD diagnosed by ultrasound or biopsy.
In keeping with these data, a study comprising 263 Columbian men found a HOMA-IR of 1.7 (AUROC 0.78 ) to be the cutoff for NAFLD diagnosed by ultrasound [31]. In a recent population-based study in Iran, the best cut-off for NAFLD diagnosed by ultrasound was 2.0 in women and 1.8 in men [32]. Even though these results in different ethnic groups seem consistent, it will be important to perform studies in ethnic groups other than Finns.

In linear regression analysis (Fig. 2), HOMA-IRs of 1.9 and 2.0 corresponded to liver fat contents of $5.0 \%$ and $5.56 \%$. The latter value is identical to that defined as the upper limit of normal liver fat measured by ${ }^{1} \mathrm{H}$-MRS in the DHS [18]. The prevalence of NAFLD in the population-based DHS was $31 \%$ [33], which is comparable with that in our cohort of research volunteers (35\%). In our cohort, the 95th percentile of liver fat in healthy individuals was $5.9 \%$. This value is close to the $5.56 \%$ in the DHS [18]. However, as our liver fat cohort was not population-based, the $5.56 \%$ in the DHS can be considered more accurate than our estimate of $5.9 \%$.

The PNPLA3 I148M variant predisposes to NAFLD but not to features of insulin resistance $[34,35]$. Thus, despite an increased liver fat content in PNPLA3 I148M variant carriers, HOMA-IR has been reported to be similar in carriers and noncarriers of similar age, sex and BMI [36-39]. Consistent with these data, in the present two healthy population-based cohorts, no difference existed in clinical characteristics between carriers and non-carriers of the PNPLA3 I148M variant. The upper limit of normal HOMA-IR was the same for both groups. In the liver fat cohort, in which $35 \%$ of individuals had NAFLD, the optimal cut-off for distinguishing NAFLD from non-NAFLD was also not affected by genotype.
Fig. 4 Linear regression between HOMA-IR measured in Helsinki by chemiluminescent immunoassay using Liaison XL (DiaSorin, Saluggia, Italy) for insulin measurement and HOMAIR measured in six other laboratories $(\mathbf{a}-\mathbf{f})$ using insulin assays as described in ESM Fig. 2. (f) Intercepts $p=0.0005$
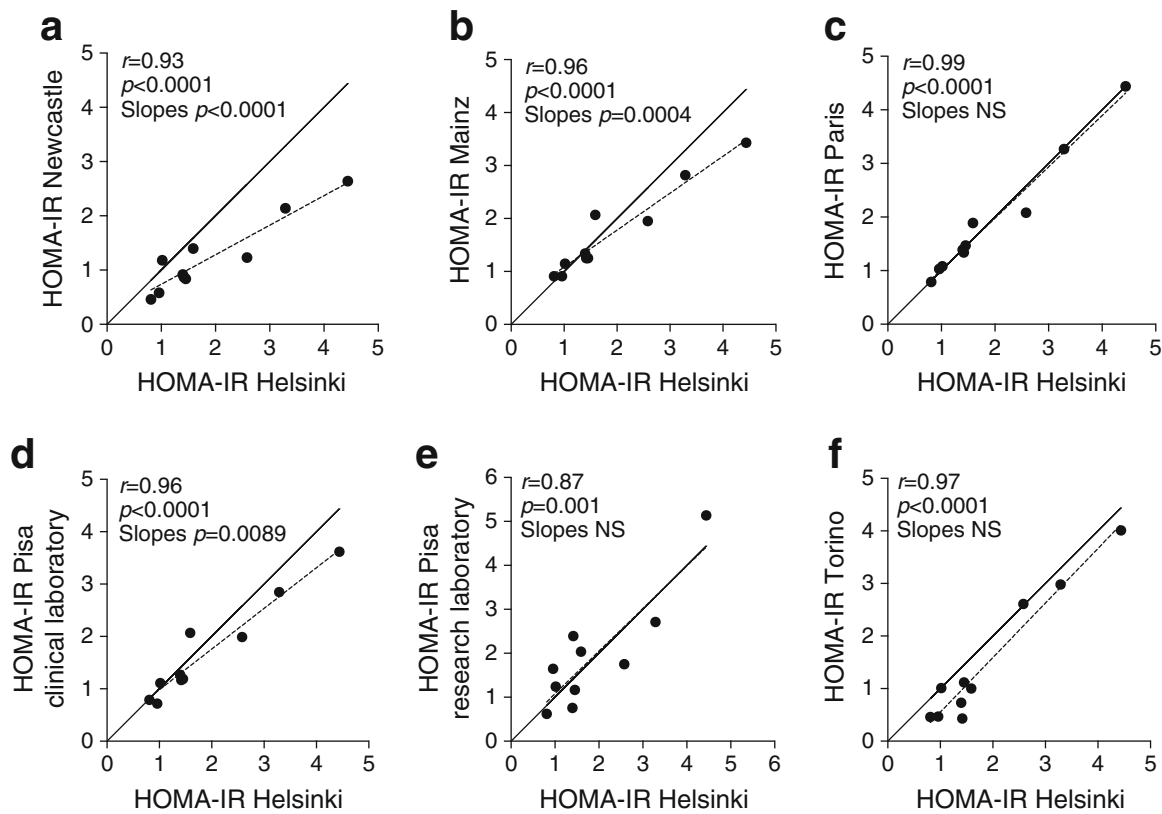
However, when comparing carriers and non-carriers at a similar liver fat content, carriers were found to have lower HOMA-IR than non-carriers (Fig. 2b). These data imply that HOMA-IR cannot be used to diagnose individuals with NAFLD due to the PNPLA3 I148M variant, and that they can only be identified by genotyping for this gene variant [10].

A limitation of HOMA-IR is that it is valid only as long as serum insulin concentrations reflect merely insulin sensitivity, not secretion [40-42]. In individuals with non-diabetic glucose tolerance, fasting glucose and insulin concentrations are closely positively correlated [43]. Once glucose tolerance becomes diabetic, insulin concentrations start to decline and their relationship to glucose is inverse rather than positive [6]. Under such conditions HOMA-IR underestimates insulin resistance-associated NAFLD, although in a recent study including 56 participants with type 2 diabetes, a HOMA-IR of 4.5 was estimated to be the optimal threshold for distinguishing NAFLD diagnosed by ultrasound or computed tomography [44]. The method used to measure insulin concentrations was not specified. The extreme example is type 1 diabetes, where there is no endogenous insulin. HOMA-IR is also influenced by insulin clearance, unlike direct measurements of insulin sensitivity. However, this may not be a problem as the decrease in insulin clearance closely parallels that in hepatic insulin sensitivity [45].

Use of HOMA-IR in the clinic assumes the degree of interlaboratory variation in insulin assays is known [29]. In the present study, we analysed fasting blood samples obtained from ten individuals covering a wide range of HOMA-IRs after a similar period of freezing and thawing and time of storage. From the regression lines relating assay results between two laboratories (Fig. 4), the upper limit of normal HOMA-IR was similar in Helsinki and Paris using the same insulin assay (2.0), but was 1.3, 1.6, 1.8, 1.8 and 2.1 in the five other laboratories using different assays. The inter-laboratory $\mathrm{CV}$ was $25 \%$. In contrast, the inter-laboratory $\mathrm{CVs}$ for other analytes, with the exception of ferritin, were much lower and ranged from $5 \%$ to $13 \%$. This implies that every laboratory should establish its own reference value for HOMA-IR, or at least understand how its insulin assay compares with other laboratories. Furthermore, reference values for HOMA-IR, even in healthy individuals, and the relationship between HOMA-IR and liver fat may be population-specific.

We conclude that the upper limit of HOMA-IR, defined based on the identification of healthy individuals in two population-based Finnish cohorts, closely corresponds to the upper limit of normal liver fat content $(<5.56 \%)$ found in the DHS. This finding supports the use of HOMA-IR in identifying individuals with 'metabolic NAFLD'. The use of HOMAIR has, however, several limitations. HOMA-IR varies considerably and more than other routine analytes among laboratories, particularly due to the use of different insulin assays. If HOMA-IR were to be used as a surrogate for insulin resistance and NAFLD, insulin assays would need to be standardised. In addition, HOMA-IR underestimates liver fat content in individuals with NAFLD associated with the PNPLA3 I148M variant and, although not examined in this study, in individuals with defective insulin secretion.

Acknowledgements We gratefully acknowledge A. Salo and A. Karioja-Kallio (Department of Medicine, Helsinki University Hospital), P. Pölönen (HUS Medical Imaging Center, Radiology, Helsinki University Hospital), M. L. Abate and C. Rosso (Department of Medical Sciences, University of Torino), M. Gaggini and E. Buzzigoli (Institute of Clinical Physiology, CNR Pisa, Italy), S. Depoix (I. Department of Medicine, University Medical Center Mainz), and D. Neeley and S. McLellen (Newcastle Hospitals NHS Foundation Trust) for skilful technical assistance; and volunteers for their help.

Data availability The data that support the findings of this study are available from the corresponding author upon reasonable request.

Funding This study was supported by research grants from the Academy of Finland (HY-J), EU/EFPIA Innovative Medicines Initiative Joint Undertaking (EMIF grant no. 115372, HY-J), the Sigrid Juselius (HY-J), the Novo Nordisk Foundations (HY-J), EVO (HY-J), The Paulo Foundation (EI) and the Finnish Medical Foundation (EI). The authors are members of the EPoS (Elucidating Pathways of Steatohepatitis) consortium, which is funded by the Horizon 2020 Framework Program of the European Union under grant agreement 634413.

Duality of interest The authors declare that there is no duality of interest associated with this manuscript.

Contribution statement $\mathrm{EI}$ and $\mathrm{YZ}$ analysed the data. EI, YZ and HY-J interpreted the data and wrote the main text. EI, HY-J, EB, KC, AG, DS and QMA designed and EI, HY-J, EB, KC, DB-R, J-ML, AG, DS, JMS and QMA acquired data in and supervised the inter-laboratory insulin assay study. AH and NL performed and interpreted the ${ }^{1} \mathrm{H}-\mathrm{MRS}$ studies. MP, PJ, SM, SK-K and JS designed, performed and supervised the population-based FINRISK/DILGOM (PJ, SM) and FIN-D2D (SK-K, JS, MP) studies. HY-J designed and supervised the project. All authors have revised the manuscript critically for important intellectual content and approved the final version of the manuscript. HY-J is the guarantor of this work.

\section{References}

1. Marchesini G, Brizi M, Bianchi G et al (2001) Nonalcoholic fatty liver disease: a feature of the metabolic syndrome. Diabetes 50: $1844-1850$

2. Bugianesi E, Gastaldelli A, Vanni E et al (2005) Insulin resistance in non-diabetic patients with non-alcoholic fatty liver disease: sites and mechanisms. Diabetologia 48:634-642

3. Matthews DR, Hosker JP, Rudenski AS et al (1985) Homeostasis model assessment: insulin resistance and beta-cell function from fasting plasma glucose and insulin concentrations in man. Diabetologia 28:412-419

4. Wallace TM, Levy JC, Matthews DR (2004) Use and abuse of HOMA modeling. Diabetes Care 27:1487-1495 
5. Radziuk J (2000) Insulin sensitivity and its measurement: structural commonalities among the methods. J Clin Endocrinol Metab 85: 4426-4433

6. Lillioja S, Mott DM, Howard BV et al (1988) Impaired glucose tolerance as a disorder of insulin action. N Engl J Med 318:1217-1225

7. Lillioja S, Mott DM, Spraul M et al (1993) Insulin resistance and insulin secretory dysfunction as precursors of non-insulindependent diabetes mellitus: prospective studies of Pima Indians. N Engl J Med 329:1988-1992

8. Defronzo RA (2009) From the triumvirate to the ominous octet: a new paradigm for the treatment of type 2 diabetes mellitus. Diabetes 58:773-795

9. Gastaldelli A (2011) Role of beta-cell dysfunction, ectopic fat accumulation and insulin resistance in the pathogenesis of type 2 diabetes mellitus. Diabetes Res Clin Pract 93:S60-S65

10. European Association for the Study of the Liver (EASL), European Association for the Study of Diabetes (EASD), European Association for the Study of Obesity (EASO) (2016) EASLEASD-EASO clinical practice guidelines for the management of non-alcoholic fatty liver disease. Diabetologia 59:1121-1140

11. Gräsbeck R, Alström T (1981) Reference values in laboratory medicine. The current state of the art. Wiley, Ann Arbor

12. Horn PS, Pesce AJ (2003) Reference intervals: an update. Clin Chim Acta 334:5-23

13. Ferrannini E, Natali A, Bell P, Cavallo-Perin P, Lalic N, Mingrone G (1997) Insulin resistance and hypersecretion in obesity. European Group for the Study of Insulin Resistance (EGIR). J Clin Invest 100:1166-1173

14. Nakai Y, Fukushima M, Nakaishi S et al (2002) The threshold value for insulin resistance on homeostasis model assessment of insulin sensitivity. Diabet Med 19:346-347

15. Geloneze B, Repetto EM, Geloneze SR, Tambascia MA, Ermetice MN (2006) The threshold value for insulin resistance (HOMA-IR) in an admixtured population IR in the Brazilian Metabolic Syndrome Study. Diabetes Res Clin Pract 72:219-220

16. Bonora E, Kiechl S, Willeit J et al (1998) Prevalence of insulin resistance in metabolic disorders: the Bruneck Study. Diabetes 47: 1643-1649

17. Manley SE, Stratton IM, Clark PM, Luzio SD (2007) Comparison of 11 human insulin assays: implications for clinical investigation and research. Clin Chem 53:922-932

18. Szczepaniak LS, Nurenberg P, Leonard D et al (2005) Magnetic resonance spectroscopy to measure hepatic triglyceride content: prevalence of hepatic steatosis in the general population. Am J Physiol Endocrinol Metab 288:E462-E468

19. Anstee QM, Day CP (2015) The genetics of nonalcoholic fatty liver disease: spotlight on PNPLA3 and TM6SF2. Semin Liver Dis 35: 270-290

20. Yki-Järvinen H (2014) Non-alcoholic fatty liver disease as a cause and a consequence of metabolic syndrome. Lancet Diabetes Endocrinol 2:901-910

21. Vartiainen E, Laatikainen T, Peltonen M et al (2010) Thirty-fiveyear trends in cardiovascular risk factors in Finland. Int J Epidemiol 39:504-518

22. Saaristo T, Peltonen M, Keinänen-Kiukaanniemi S et al (2007) National type 2 diabetes prevention programme in Finland: FIND2D. Int J Circumpolar Health 66:101-112

23. Kotronen A, Peltonen M, Hakkarainen A et al (2009) Prediction of non-alcoholic fatty liver disease and liver fat using metabolic and genetic factors. Gastroenterology 137:865-872

24. Friedewald WT, Levy RI, Fredrickson DS (1972) Estimation of the concentration of low-density lipoprotein cholesterol in plasma, without use of the preparative ultracentrifuge. Clin Chem 18:499-502

25. Kotronen A, Johansson LE, Johansson LM et al (2009) A common variant in PNPLA3, which encodes adiponutrin, is associated with liver fat content in humans. Diabetologia 52:1056-1060
26. Männistö S, Harald K, Kontto J et al (2014) Dietary and lifestyle characteristics associated with normal-weight obesity: the National FINRISK 2007 Study. Br J Nutr 111:887-894

27. Hyysalo J, Männistö VT, Zhou Y et al (2014) A population-based study on the prevalence of NASH using scores validated against liver histology. J Hepatol 60:839-846

28. Greiner M, Pfeiffer D, Smith RD (2000) Principles and practical application of the receiver-operating characteristic analysis for diagnostic tests. Prev Vet Med 45:23-41

29. Manley SE, Luzio SD, Stratton IM, Wallace TM, Clark PMS (2008) Preanalytical, analytical, and computational factors affect homeostasis model assessment estimates. Diabetes Care 31:1877-1883

30. Salgado AL, Carvalho LD, Oliveira AC, Santos VN, Vieira JG, Parise ER (2010) Insulin resistance index (HOMA-IR) in the differentiation of patients with non-alcoholic fatty liver disease and healthy individuals. Arq Gastroenterol 47:165-169

31. Perez M, Gonzáles L, Olarte R et al (2011) Nonalcoholic fatty liver disease is associated with insulin resistance in a young Hispanic population. Prev Med 52:174-177

32. Motamed N, Miresmail SJH, Rabiee B et al (2016) Optimal cutoff points for HOMA-IR and QUICKI in the diagnosis of metabolic syndrome and non-alcoholic fatty liver disease: a population based study. J Diabetes Complicat 30:269-274

33. Browning JD, Szczepaniak LS, Dobbins R et al (2004) Prevalence of hepatic steatosis in an urban population in the United States: impact of ethnicity. Hepatology 40:1387-1395

34. Sookoian S, Pirola CJ (2011) Meta-analysis of the influence of I148M variant of patatin-like phospholipase domain containing 3 gene (PNPLA3) on the susceptibility and histological severity of nonalcoholic fatty liver disease. Hepatology 53:1883-1894

35. Petäjä EM, Yki-Järvinen H (2016) Definitions of normal liver fat and the association of insulin sensitivity with acquired and genetic NAFLD — a systematic review. Int J Mol Sci 17:E633

36. Verrijken A, Beckers S, Francque S et al (2013) A gene variant of PNPLA3, but not of APOC3, is associated with histological parameters of NAFLD in an obese population. Obesity 21:2138-2145

37. Romeo S, Sentinelli F, Cambuli VM et al (2010) The 148M allele of the PNPLA3 gene is associated with indices of liver damage early in life. J Hepatol 53:335-338

38. Valenti L, Alisi A, Galmozzi E et al (2010) I148M patatin-like phospholipase domain-containing 3 gene variant and severity of pediatric nonalcoholic fatty liver disease. Hepatology 52:12741280

39. Del Ben M, Polimeni L, Brancorsini M et al (2014) Non-alcoholic fatty liver disease, metabolic syndrome and patatin-like phospholipase domain-containing protein3 gene variants. Eur J Intern Med 25:566-570

40. Singh B, Saxena A (2010) Surrogate markers of insulin resistance: a review. World J Diabetes 1:36-47

41. Borai A, Livingstone C, Farzal A, Kholeif M, Wang T, Ferns G (2010) Reproducibility of HOMA and QUICKI among individuals with variable glucose tolerance. Diabetes Metab 36:247-249

42. Bonora E, Targher G, Alberiche M et al (2000) Homeostasis model assessment closely mirrors the glucose clamp technique in the assessment of insulin sensitivity: studies in subjects with various degrees of glucose tolerance and insulin sensitivity. Diabetes Care 23: $57-63$

43. Defronzo RA, Bonadonna RC, Ferrannini E (1992) Pathogenesis of NIDDM: a balanced overview. Diabetes Care 15:318-368

44. Gutierrez-Buey G, Núñez-Córdoba JM, Llavero-Valero M, Gargallo J, Salvador J, Escalada J (2017) Is HOMA-IR a potential screening test for non-alcoholic fatty liver disease in adults with type 2 diabetes? Eur J Intern Med. doi:10.1016/j.ejim.2017.03.006

45. Kotronen A, Vehkavaara S, Seppälä-Lindroos A et al (2007) Effect of liver fat on insulin clearance. Am J Physiol Endocrinol Metab 293:E1709-E1715 